\title{
119Sb--a potent auger emitter for targeted radionuclide therapy
}

Thisgaard, H.; Jensen, Kai Mikael

Published in:

Medical Physics

DOI:

10.1118/1.2963993

Publication date:

2008

Document version

Publisher's PDF, also known as Version of record

Citation for published version (APA):

Thisgaard, H., \& Jensen, K. M. (2008). ${ }^{119}$ Sb--a potent auger emitter for targeted radionuclide therapy. Medical Physics, 35(9), 3839-3846. https://doi.org/10.1118/1.2963993 


\title{
${ }^{119} \mathrm{Sb}-\mathrm{A}$ potent Auger emitter for targeted radionuclide therapy
}

\author{
$\mathrm{H}$. Thisgaard ${ }^{\mathrm{a})}$ and $\mathrm{M}$. Jensen ${ }^{\mathrm{b})}$ \\ The Hevesy Laboratory, Radiation Research Department, Risoe National Laboratory for Sustainable \\ Energy, Technical University of Denmark, P.O. 49, DK-4000 Roskilde, Denmark; and Department of Natural \\ Sciences, Faculty of Life Sciences, University of Copenhagen, DK-1871 Frederiksberg, Denmark
}

(Received 17 March 2008; revised 2 July 2008; accepted for publication 2 July 2008; published 7 August 2008)

\begin{abstract}
Auger electron emitting radionuclides in cancer therapy offer the opportunity to deliver a high radiation dose to the tumor cells with high radiotoxicity while minimizing toxicity to normal tissue. We have in this study identified the Auger emitter ${ }^{119} \mathrm{Sb}$ as a potent nuclide for targeted radionuclide therapy based on theoretical dosimetry calculations at a subcellular scale. From these calculations we have determined the cellular S-values for this therapeutic isotope. Moreover, we have demonstrated the possibility of producing this isotope and also the SPECT-analogue ${ }^{117} \mathrm{Sb}$ for patientspecific dosimetry, by measuring the proton irradiation yields for both isotopes using a low-energy cyclotron. The excellent SPECT imaging properties of the ${ }^{117} \mathrm{Sb}$ radionuclide have been shown by scanning a Jaszczak SPECT Phantom. (c) 2008 American Association of Physicists in Medicine. [DOI: $10.1118 / 1.2963993]$
\end{abstract}

Key words: Auger electrons, Sb-119, S-values, radionuclide therapy, Sb-117, cancer, production yield, SPECT, planar scintigraphy, Jaszczak phantom

\section{INTRODUCTION}

In recent years the use of Auger electron emitting radionuclides for targeted radionuclide therapy has shown promising results. ${ }^{1-7}$ The low-energetic Auger electrons including the even lower energetic Coster-Kronig (CK) electrons are emitted by isotopes that decay by electron capture (EC) or have internal conversion (IC) in their decay. Depending on the isotope, many such electrons can be emitted per decay (from a few to more than 35$)^{8}$ almost instantaneously, thus, creating an electron cascade. The multiplicity and the short range in tissue (from a few nm to some $\mu \mathrm{m}$ ) of these Auger and CK electrons give rise to a high energy density created in the immediate vicinity of the decay site and, thus, a high, very localized absorbed radiation dose to the target region. ${ }^{9}$ Additionally, the short range minimizes irradiation of the neighboring, normal cells, resulting in low observed unspecific radiotoxicities in cell experiments. ${ }^{3,10}$ The very short range of the electrons makes the localization of the radionuclides with respect to the sensitive targets in the cells (DNA) critical in determining effects from the radiation. If the decaying radionuclides are incorporated directly into the nuclear DNA or in close proximity to the DNA, extreme radiotoxicity is observed, resembling high-LET radiation with relative biological effectiveness (RBE) values much higher than $1 .^{11,12}$ On the other hand, if the radionuclides are bound outside the cellular nucleus, e.g., on the cellular membrane, located in the cytoplasm or extracellular, the effects resemble those observed with low-LET radiation (e.g., X-rays) with low RBE values.

Some of the most commonly used Auger emitters for research in radionuclide therapy are ${ }^{125} \mathrm{I},{ }^{123} \mathrm{I},{ }^{111} \mathrm{In},{ }^{67} \mathrm{Ga}$, and ${ }^{201} \mathrm{Tl}$, which are all readily obtainable from commercial sources - the last four isotopes due to their wide use in diagnostics tracers (SPECT) in nuclear medicine. ${ }^{3,4,13-15}$ How- ever, the wide use of these isotopes in research in the field of radionuclide therapy is probably more a consequence of this accessibility than because of optimal radiation physics properties for cancer therapy.

In principle, the optimal radionuclide for targeted radiotherapy must be one that, in addition to a proper half-life (hours $\rightarrow$ days), is emitting radiation with a range that is long enough to allow irradiation of the target region, but at the same time, short enough to spare healthy tissue surrounding this region. Thus, a high proportion of gamma emission as seen with the four SPECT isotopes mentioned above is an undesirable property. For dosimetry calculations, however, the emission of gamma radiation is useful to determine the time-activity curve for the administered radiopharmaceutical in, e.g., the critical organs but the gamma intensity should be low to minimize unwanted dose to healthy tissues. Alternatively, the isotope used for therapy could emit no penetrating radiation and the time-activity curve and pharmacokinetics could then be evaluated before the treatment starts or simultaneously from a tracer study with a SPECT- or a PETisotope of the same element as the one used for the therapy. Examples of such isotope pairs are ${ }^{86} \mathrm{Y} /{ }^{90} \mathrm{Y}$ and ${ }^{124} \mathrm{I} /{ }^{131} \mathrm{I} .{ }^{16-20}$

There could be a need for increasing the scarce selection of available radionuclides suitable for targeted radionuclide therapy. In this study, we have performed a comparison of several Auger emitters based on theoretical dosimetry calculations at subcellular and macroscopic levels. From this we conclude that the radionuclide ${ }^{119} \mathrm{Sb}\left(\mathrm{T}_{1 / 2}=38.19 \mathrm{~h}\right)$ is a potent Auger-electron emitter that possibly can be used in therapy of small metastasis and disseminated cancer cells. The radionuclide ${ }^{117} \mathrm{Sb}\left(\mathrm{T}_{1 / 2}=2.8 \mathrm{~h}\right)$ can give SPECT- or SPECT/CT-based patient-specific 3D dosimetry. 


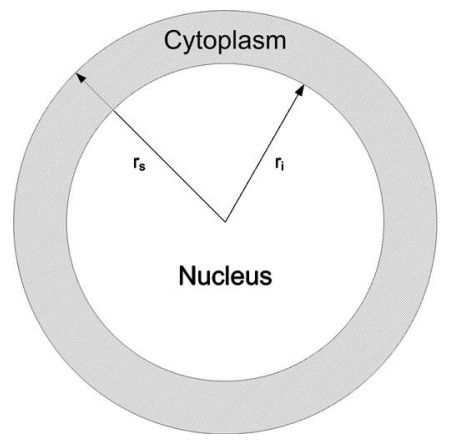

This paper presents the first attempt to our knowledge to evaluate ${ }^{119} \mathrm{Sb}$ for subcellular radioactivity distributions with the calculation of the cellular S-values for this isotope. As these results are very encouraging, we have measured the proton irradiation yields for ${ }^{119} \mathrm{Sb}$ and ${ }^{117} \mathrm{Sb}$ on enriched target materials. Moreover, we have demonstrated that ${ }^{117} \mathrm{Sb}$ is very suitable for SPECT imaging by scanning a Jaszczak SPECT phantom.

\section{MATERIALS AND METHODS}

\section{II.A. Dose calculations and cellular S-values}

The cellular dosimetry was performed using the formalism described in MIRD Cellular S-values ${ }^{21}$ with a code programmed in MatLab 7.0.1. Using the geometric reduction factors by Berger $^{22}$ (Fig. 1) and stopping powers obtained from an empirical energy-loss expression using the continuous-slowing down approximation (CSDA), the dose per cumulated decay to all parts of the tumor cell could be calculated (Figs. 3-5). The energy-loss expression, i.e., the relationship between electron energy $(E)$ and range $(R)$ in unit density matter was taken as experimentally determined by Cole for energies higher than $0.4 \mathrm{keV} .^{23}$ Below $0.4 \mathrm{keV}$, a fit was made to Cole's experimental data because these are not well described by Cole's expression. The following expression was used: $E=-53111 R^{3}+1491.4 R^{2}+11.893 R$, with $E$ in $\mathrm{keV}$ and $R$ in $\mu \mathrm{m}$. Using this approach, it is assumed that secondary electrons (delta rays) are absorbed locally. In calculating the S-values, this assumption has been shown to be valid from comparison with Monte Carlo transport codes for electron energies below several hundred $\mathrm{keV}$. $^{21}$

The radioactivity in the cell was assumed uniformly distributed over one of the following spherical symmetric cell compartments: the cell surface (CS), cytoplasm (Cy), or cell nucleus $(\mathrm{N})$. As target region, only the cell nucleus was considered in calculating the S-values. The contribution to the cellular absorbed mean dose from gamma radiations was not taken into account since this is negligible as reported in MIRD Cellular S-values. ${ }^{21}$

The radiation spectra were either obtained from the Report No. 2 of AAPM Nuclear Task Group No. 6 by Howell, ${ }^{8}$ if possible, or from the Nuclear Decay Data Files for Dose Calculation (DECDC). ${ }^{24}$ The spectra from DECDC contain data for the K-, L-, and M-shell electrons, while the spectra reported by Howell are more complete by including the low- energy $\mathrm{N}$ - and O-shell electrons. The omission of these very low-energy electrons was seen to be insignificant for sphere diameters larger than $1 \mu \mathrm{m}$ in agreement with the findings reported by Howell. ${ }^{8}$

In calculating the tumor-to-normal-tissue dose ratios (TNDs) per disintegration, the dose to the normal tissue was obtained from the tabulated dose factors [(DFs), equivalent to the MIRD organ S-values], in the Radiation dose assessment resource (RADAR). ${ }^{25}$ Only the whole-body to wholebody dose contribution was considered, i.e., the $\mathrm{S}$ (whole-body $\leftarrow$ whole-body) assuming a uniform activity distribution in this compartment. The TND calculation was done on a simple per decay basis, i.e., differences in physical and biological half-lives and resulting differences in cumulated decays were not taken into account at this stage.

\section{II.B. ${ }^{119} \mathrm{Sb}$ and ${ }^{117} \mathrm{Sb}$ productions}

As the above calculations identify ${ }^{119} \mathrm{Sb}$ as a potent isotope for radionuclide therapy, the proton irradiation yields of this isotope and its SPECT-analogue ${ }^{117} \mathrm{Sb}$ were measured.

\section{II.B.1. Target preparation}

The production of the ${ }^{119} \mathrm{Sb}$ and ${ }^{117} \mathrm{Sb}$ radionuclides was performed via the nuclear reactions: ${ }^{119} \mathrm{Sn}(\mathrm{p}, \mathrm{n}){ }^{119} \mathrm{Sb}$ and ${ }^{117} \mathrm{Sn}(\mathrm{p}, \mathrm{n}){ }^{117} \mathrm{Sb}$, respectively. Before the irradiations, each enriched tin target (enrichment: $97.4 \%{ }^{119} \mathrm{Sn}$ and $97.6 \%$

${ }^{117} \mathrm{Sn}$, respectively, both from Campro Scientific) was made by either dissolving the tin metal $(5-30 \mathrm{mg})$ directly in hot $2.5 \mathrm{M} \mathrm{KOH}$ containing $\mathrm{H}_{2} \mathrm{O}_{2}$ or by dissolving the tin metal in $0.5 \mathrm{ml}$ hot, concentrated $\mathrm{HCl}$ containing $\mathrm{H}_{2} \mathrm{O}_{2}$ followed by adding $1.0 \mathrm{ml} 10 \mathrm{M} \mathrm{KOH}$ to the solution. The latter route was used for larger quantities of the tin metal to speed up the etching process. The resulting solution was then diluted to $0.25 \mathrm{M} \mathrm{KOH}$ with distilled water and transferred to an electroplating cell. A coin-like silver plate with a diameter of $29 \mathrm{~mm}$ and thickness of $5.2 \mathrm{~mm}$ was used as backing (see Fig. 2). The electroplating process was carried out with a bath temperature of approximately $65-70{ }^{\circ} \mathrm{C}$ with a plating current density of $4-6 \mathrm{~mA} / \mathrm{cm}^{2}$ for $6-8 \mathrm{~h}$. The target thicknesses of the ${ }^{117} \mathrm{Sn}$ and ${ }^{119} \mathrm{Sn}$ targets were determined from the weight and surface area of the electroplated tin to be 7.4 and $5.5 \mathrm{mg} / \mathrm{cm}^{2}$, respectively. 


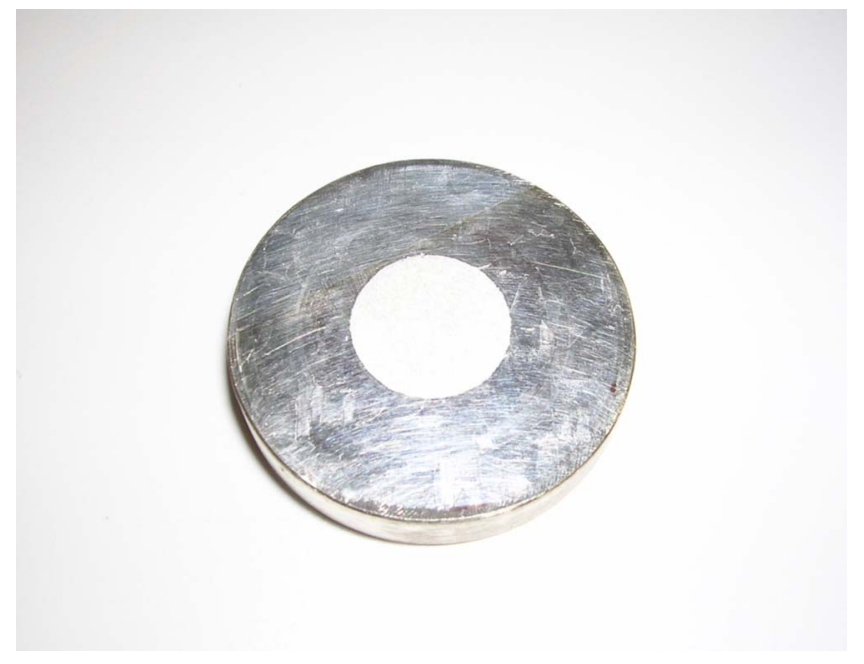

FIG. 2. Electroplated tin target $(\varnothing 10 \mathrm{~mm})$ on a silver backing $(\varnothing 29 \mathrm{~mm}$, $5.2 \mathrm{~mm}$ thickness).

\section{II.B.2. Irradiations and activity measurements}

The electroplated targets were mounted in a water cooled irradiation chamber with an aluminium proton energy degrader in front of the target. The irradiations were done using the external proton beam from the beamline of the GE PETtrace Cyclotron at the Hevesy Laboratory at Risoe National Laboratory. The targets were irradiated with a constant beam current of $15 \mu \mathrm{A}$ with a collimated beam of $10 \mathrm{~mm}$ in diam for $1 \mathrm{~h}$. The integrated beam current was taken as reported by the cyclotron software. The proton energy was determined from prior irradiations by irradiating a precision $\mathrm{Cu}$ monitor foil. The energy was measured to be $10.1 \mathrm{MeV}$ behind the energy degrader using the monitor reactions ${ }^{\text {nat }} \mathrm{Cu}(\mathrm{p}, \mathrm{x}){ }^{63} \mathrm{Zn}$ and ${ }^{\text {nat }} \mathrm{Cu}(\mathrm{p}, \mathrm{x}){ }^{62} \mathrm{Zn}$ with the recommended cross sections given by IAEA. ${ }^{26}$

The produced ${ }^{117} \mathrm{Sb}$ activity was measured using a calibrated Ge detector (Princeton Gamma-Tech, LGC 5) with the detector software Genie 2000 (version 3.0). The energy and efficiency calibrations were done using ${ }^{152} \mathrm{Eu}$ and ${ }^{133} \mathrm{Ba}$ point sources (AEA Technology). The irradiated target was measured several times with a distance ranging between $20 \mathrm{~cm}$ and $300 \mathrm{~cm}$ from the detector in the time interval from $1 \mathrm{~h}$ to 4 days after EOB. The ${ }^{117} \mathrm{Sb}$ activity was determined primarily from the $158.56 \mathrm{keV} \gamma$-ray $\left(I_{\gamma}: 86 \%\right) .{ }^{27} \mathrm{By}$ measuring the target after the ${ }^{117} \mathrm{Sb}$ had decayed, it was possible to correct the ${ }^{117} \mathrm{Sb}$ activity for any simultaneously produced ${ }^{117 m} \mathrm{Sn}\left(\mathrm{T}_{1 / 2}=13.60 \mathrm{~d}\right)$, which emits a $\gamma$-ray of the same energy $(158.56 \mathrm{keV})$.

For determining the produced ${ }^{119} \mathrm{Sb}$ activity, a low-energy Ge detector (Canberra, GL0055P) was used. This setup made it possible to discriminate the $23.87 \mathrm{keV} \gamma$-ray stemming from the ${ }^{119} \mathrm{Sb}$ decay from the characteristic X-rays simultaneously emitted from both the ${ }^{119} \mathrm{Sb}$ and impurities with EC or IC decay, if present. The energy and efficiency calibrations were done using ${ }^{241} \mathrm{Am}$ and ${ }^{210} \mathrm{~Pb}$ point sources (AEA Technology). The ${ }^{119} \mathrm{Sb}$ activity was calculated from the $23.87 \mathrm{keV} \gamma$-ray $\left(I_{\gamma}: 16.1 \%\right)^{27}$ using Genie 2000. The target was measured several times with a distance of $39 \mathrm{~cm}$ from the detector in the time interval from $52 \mathrm{~h}$ to several weeks after EOB. The long delay between EOB and the first measurement was in order to let the simultaneously produced ${ }^{107} \mathrm{Cd}$ in the silver backing decay. The ${ }^{119} \mathrm{Sb}$ activity was corrected for any simultaneously produced ${ }^{119 m} \mathrm{Sn}\left(\mathrm{T}_{1 / 2}\right.$ $=293.1 \mathrm{~d}$ ), which emits a single $\gamma$-ray of the same energy $(23.87 \mathrm{keV})$, by measuring the target after the ${ }^{119} \mathrm{Sb}$ had decayed. The effective attenuation of the $23.87 \mathrm{keV} \gamma$-ray in the "thick" ${ }^{119}$ Sn layer was calculated to be $4 \%$ and this correction was included in the activity measurement.

\section{II.C. ${ }^{117}$ Sb SPECT}

To demonstrate the imaging capabilities of the ${ }^{117} \mathrm{Sb}$ isotope, a planar scintigraphy and a single photon emission computed tomography (SPECT) scan was made of a Jaszczak SPECT phantom.

\section{II.C.1. ${ }^{117} \mathrm{Sb}$ production}

The enriched ${ }^{117} \mathrm{Sn}$ target used in the yield measurements was irradiated using the setup described above with a $15 \mu \mathrm{A}$ proton beam (14.9 $\mu \mathrm{A}$ mean current) for $90 \mathrm{~min}$. After the

TABLE I. The calculated S-values for varying cell sizes with ${ }^{119} \mathrm{Sb}$ activity uniformly distributed in one of the following cell compartments: $\mathrm{N}$ : Nucleus, Cy: Cytoplasm, or CS: Cell Surface.

\begin{tabular}{|c|c|c|c|c|}
\hline \multicolumn{2}{|c|}{ Cell size $[\mu \mathrm{m}]$} & \multicolumn{3}{|c|}{ S-values $[\mathrm{Gy} /(\mathrm{Bq} \mathrm{s})]$} \\
\hline$r_{C}$ & $r_{N}$ & $\mathrm{~S}(\mathrm{~N} \leftarrow \mathrm{N})$ & $\mathrm{S}(\mathrm{N} \leftarrow \mathrm{Cy})$ & $\mathrm{S}(\mathrm{N} \leftarrow \mathrm{CS})$ \\
\hline 3 & 1 & $2.44 \times 10^{-1}$ & $5.50 \times 10^{-3}$ & $2.11 \times 10^{-3}$ \\
\hline 3 & 2 & $3.72 \times 10^{-2}$ & $4.16 \times 10^{-3}$ & $2.27 \times 10^{-3}$ \\
\hline 4 & 2 & $3.72 \times 10^{-2}$ & $2.51 \times 10^{-3}$ & $1.32 \times 10^{-3}$ \\
\hline 4 & 3 & $1.28 \times 10^{-2}$ & $2.23 \times 10^{-3}$ & $1.44 \times 10^{-3}$ \\
\hline 5 & 2 & $3.72 \times 10^{-2}$ & $1.77 \times 10^{-3}$ & $9.19 \times 10^{-4}$ \\
\hline 5 & 3 & $1.28 \times 10^{-2}$ & $1.56 \times 10^{-3}$ & $9.77 \times 10^{-4}$ \\
\hline 5 & 4 & $6.16 \times 10^{-3}$ & $1.45 \times 10^{-3}$ & $1.07 \times 10^{-3}$ \\
\hline 6 & 3 & $1.28 \times 10^{-2}$ & $1.22 \times 10^{-3}$ & $7.87 \times 10^{-4}$ \\
\hline 6 & 4 & $6.16 \times 10^{-3}$ & $1.12 \times 10^{-3}$ & $7.82 \times 10^{-4}$ \\
\hline 6 & 5 & $3.60 \times 10^{-3}$ & $1.03 \times 10^{-3}$ & $7.87 \times 10^{-4}$ \\
\hline 7 & 3 & $1.28 \times 10^{-2}$ & $1.02 \times 10^{-3}$ & $6.31 \times 10^{-4}$ \\
\hline 7 & 4 & $6.16 \times 10^{-3}$ & $9.18 \times 10^{-4}$ & $5.87 \times 10^{-4}$ \\
\hline 7 & 5 & $3.60 \times 10^{-3}$ & $8.16 \times 10^{-4}$ & $5.67 \times 10^{-4}$ \\
\hline 7 & 6 & $2.34 \times 10^{-3}$ & $7.33 \times 10^{-4}$ & $5.70 \times 10^{-4}$ \\
\hline 8 & 4 & $6.16 \times 10^{-3}$ & $7.62 \times 10^{-4}$ & $4.35 \times 10^{-4}$ \\
\hline 8 & 5 & $3.60 \times 10^{-3}$ & $6.71 \times 10^{-4}$ & $4.16 \times 10^{-4}$ \\
\hline 8 & 6 & $2.34 \times 10^{-3}$ & $5.88 \times 10^{-4}$ & $4.08 \times 10^{-4}$ \\
\hline 8 & 7 & $1.62 \times 10^{-3}$ & $5.28 \times 10^{-4}$ & $4.14 \times 10^{-4}$ \\
\hline 9 & 4 & $6.16 \times 10^{-3}$ & $6.33 \times 10^{-4}$ & $3.09 \times 10^{-4}$ \\
\hline 9 & 5 & $3.60 \times 10^{-3}$ & $5.57 \times 10^{-4}$ & $3.00 \times 10^{-4}$ \\
\hline 9 & 6 & $2.34 \times 10^{-3}$ & $4.86 \times 10^{-4}$ & $2.95 \times 10^{-4}$ \\
\hline 9 & 7 & $1.62 \times 10^{-3}$ & $4.27 \times 10^{-4}$ & $2.97 \times 10^{-4}$ \\
\hline 9 & 8 & $1.17 \times 10^{-3}$ & $3.88 \times 10^{-4}$ & $3.07 \times 10^{-4}$ \\
\hline 10 & 5 & $3.60 \times 10^{-3}$ & $4.62 \times 10^{-4}$ & $2.06 \times 10^{-4}$ \\
\hline 10 & 6 & $2.34 \times 10^{-3}$ & $4.04 \times 10^{-4}$ & $2.09 \times 10^{-4}$ \\
\hline 10 & 7 & $1.62 \times 10^{-3}$ & $3.55 \times 10^{-4}$ & $2.13 \times 10^{-4}$ \\
\hline 10 & 8 & $1.17 \times 10^{-3}$ & $3.16 \times 10^{-4}$ & $2.20 \times 10^{-4}$ \\
\hline 10 & 9 & $8.74 \times 10^{-4}$ & $2.92 \times 10^{-4}$ & $2.32 \times 10^{-4}$ \\
\hline
\end{tabular}




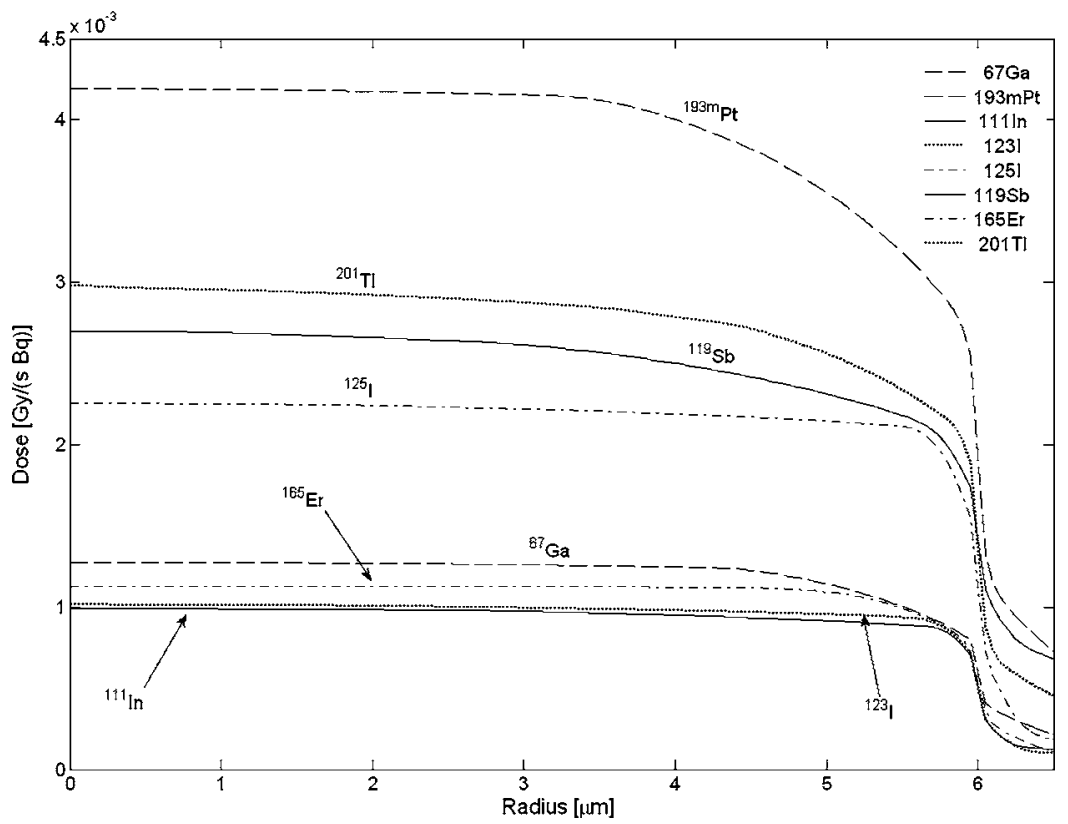

FIG. 3. The dose to various parts of a cell (nuclear radius: $6 \mu \mathrm{m}$, cellular radius: $8 \mu \mathrm{m}$ ) per cumulated decay as a function of radius for a uniform activity distribution in the cell nucleus.

irradiation, the target material was dissolved with hot conc. $\mathrm{HCl}$ with $\mathrm{H}_{2} \mathrm{O}_{2}$ added to oxidize the strong reductant, $\mathrm{Sn}$ (II), to $\mathrm{Sn}(\mathrm{IV})$. This speeds up the etching of the Sn and avoids reduction of the dissolved $\mathrm{Sb}(\mathrm{III})$ to $\mathrm{Sb}(0)$ on the silver backing. After the produced ${ }^{117} \mathrm{Sb}$ was dissolved, the radionuclidic purity of the resulting solution was measured using the Ge detector (Princeton Gamma-Tech, LGC 5) described above. Because no radioactive tin impurities were detected in the solution, no attempt was made to separate the tin and the produced radioantimony.

\section{II.C.2. Planar scintigraphy and SPECT}

The planar scintigraphy (PS) and the SPECT scan were both made using the Jaszczak SPECT phantom. The PS was made of the phantom with the cold rod insert only. The phantom was filled with water containing $202 \mathrm{MBq}{ }^{117} \mathrm{Sb}$ to a height of $8.8 \mathrm{~cm}$, i.e., to just above the cold rod insert. The phantom was placed on the collimator face of one of the detectors of a Philips SKYLight dual-head gamma camera with medium energy general purpose (MEGP) collimators mounted. A total of 10 million counts were acquired.

The SPECT scan was made with the phantom completely filled with water containing $153 \mathrm{MBq}$ of ${ }^{117} \mathrm{Sb}$. This time, both the cold rod insert and the six solid spheres (cold spots) were mounted in the phantom. To simulate a hot spot, we additionally mounted a small cylindrical vial $(\varnothing 10 \mathrm{~mm}$, height $25 \mathrm{~mm}$ ) filled with water containing $15 \mathrm{MBq}$ of ${ }^{117} \mathrm{Sb}$ offcenter in the volume above the cold rod insert. The phantom was then placed on its side on the bed and scanned. The acquisition consisted of 32 frames of $30 \mathrm{sec}$ with the MEGP collimators mounted on the cameras.

The image reconstruction was performed using filtered backprojection with a Hanning filter followed by standard attenuation correction. All images were then transferred to MatLab 7.0.1 for noise reduction and background subtraction.

\section{RESULTS AND DISCUSSION}

\section{III.A. Cellular S-values and TND}

The calculated cellular S-values for ${ }^{119} \mathrm{Sb}$ activity located on the cell surface, in the cytoplasm, or in the cell nucleus, respectively, for different sizes of cells and cell nuclei can be seen in Table I. Plots showing the dose per disintegration $[\mathrm{Gy} /(\mathrm{Bq} \mathrm{s})]$ as a function of radius for activity in the three compartments for several different radionuclides are shown in Figs. 3-5. These plots are for illustrations calculated for cells with a radius of $8 \mu \mathrm{m}$ and a nucleus radius of $6 \mu \mathrm{m}$-radii taken for being characteristic values for tumor cells. Of course, these values can vary between cell populations and tumor cell types.

For radioactivity bound to the cell membrane or distributed uniformly in the cytoplasm, it can be seen that ${ }^{119} \mathrm{Sb}$ clearly delivers the highest dose to the cell nucleus of the radionuclides tested. The results for the cell membrane bound activity are in agreement with those reported by Sastry et al. ${ }^{28}$ who calculated that the $20 \mathrm{keV}$ conversion and $\mathrm{K}$-Auger electrons from ${ }^{119} \mathrm{Sb}$ are optimal for irradiating the nuclei of cells from radiolabeled monoclonal antibodies bound to the cell surface. For radioactivity distributed in the cell nucleus, ${ }^{119} \mathrm{Sb}$ delivers a high dose compared to most of the radionuclides tested - it is only exceeded by ${ }^{201} \mathrm{Tl}$ and ${ }^{193 m} \mathrm{Pt}$.

However, in radionuclide therapy, the effect of a given treatment is usually limited by the absorbed dose to the critical organs, i.e., the dose-limiting organs and, thus, it is necessary to include the normal tissue dose. ${ }^{29,30}$ For very small tumors and micrometastases with Auger-emitters located in the cell nuclei, the self-dose is dominating ${ }^{31}$ and the crossdose contribution may then be neglected. Consequently, it was possible to calculate a tumor-to-normal-tissue dose ratio (TND) per disintegration for decays in the tumor cell nuclei and decays in the total body as described in Sec. II A. The 


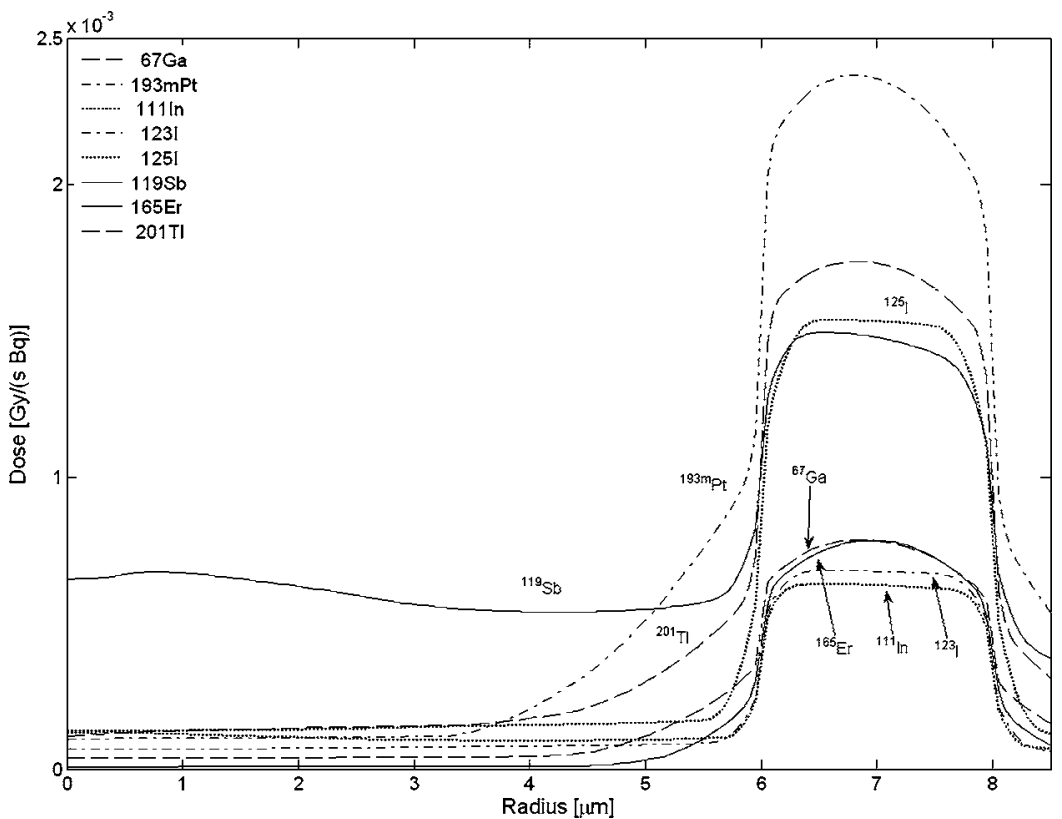

FIG. 4. The dose to various parts of a cell (nuclear radius: $6 \mu \mathrm{m}$, cellular radius: $8 \mu \mathrm{m}$ ) per cumulated decay as a function of radius for a uniform activity distribution in the cell cytoplasm. results for different nuclides normalized to the TND value for ${ }^{119} \mathrm{Sb}$ for cells with a radius of $8 \mu \mathrm{m}$ and a nucleus radius of $6 \mu \mathrm{m}$ can be seen in Fig. 6 .

From Fig. 6 it can be seen that despite the high absorbed doses delivered to the tumor cell nucleus per disintegration from ${ }^{201} \mathrm{Tl}$ and ${ }^{193 m} \mathrm{Pt}$ (Fig. 3), their TND values are low compared to some of the other isotopes tested. This is due to the abundance of relatively high energy conversion electrons (above 50-100 keV) emitted in the decays of these isotopes with ranges up to more than $0.2 \mathrm{~mm}$ in tissue ${ }^{8}$ in addition to the abundant photon emission ( $\gamma$ - and especially X-rays) from the ${ }^{201} \mathrm{Tl}$ decay. Based on a theoretical study of different isotopes located in varying sizes of spherical tumors in a patient (modeled as an ellipsoid), Bernhardt et al. concluded that to achieve a high TND in the treatment of small tumors
$(<1000$ cells), the energy of the emitted electrons should be $\leqslant 40 \mathrm{keV} .{ }^{29}$ In addition, the photon-to-electron energy emission ratio, $p / e$, should be $\leqslant 2$ (the $p / e$ of ${ }^{201} \mathrm{Tl}$ is 2.1 ). These statements are in agreement with our findings. For heterogeneous radioactivity distributions, which are often seen in larger tumors, the cross-irradiation component resulting from these high energy conversion electrons would result in a more uniform dose distribution, though, and, thus, could be an advantage. However, it has been shown that in therapy of neuroendocrine tumors with the Auger- and conversion electron emitter ${ }^{111}$ In as ${ }^{111}$ In-DTPA-Octreotide, it is the Auger electrons and not the conversion electrons (145-245 keV) that are responsible for the observed therapeutic effects. ${ }^{2}$

The normalized TND values for activity distributed in the cytoplasm or on the cell membrane can also be seen in Fig.

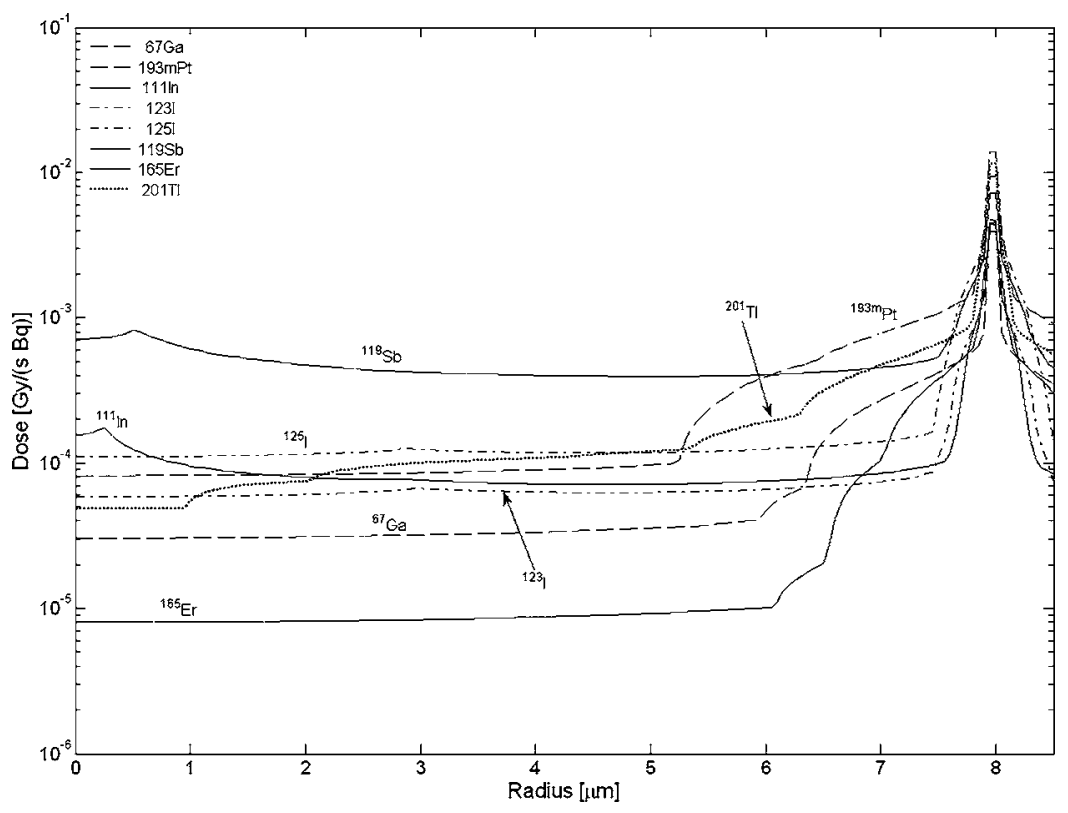

FIG. 5. The dose to various parts of a cell (nuclear radius: $6 \mu \mathrm{m}$, cellular radius: $8 \mu \mathrm{m}$ ) per cumulated decay as a function of radius for a uniform activity distribution on the cell surface. 


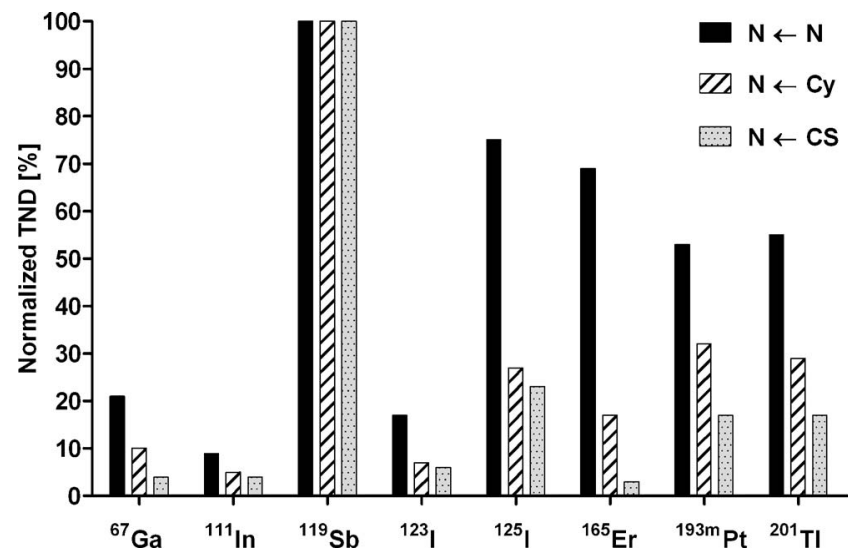

FIG. 6. Calculated tumor-to-normal-tissue dose ratios (TNDs) per disintegration for decays in different tumor cell compartments $(\mathrm{N}$ : nucleus, $\mathrm{Cy}$ : cytoplasm, or CS: cell surface) and decays in the total body (normal tissue). The results are normalized to the corresponding TND values for ${ }^{119} \mathrm{Sb}$.

6. These results are only valid for single cells or very small cell clusters consisting of a low number of cells due to the omission of the cross-dose contribution, which starts to account for a considerable part of the total tumor cell dose when the cluster size increases. ${ }^{31}$ In order to calculate the TND values for larger cell clusters, both the cluster size, the cluster and cell geometries, and the activity distributions have to be taken into account. Since the rationale of using Auger electrons for cancer therapy is to be able to exploit the possible high-LET-like biological effects, so far only obtained from nuclear localization of the Auger emitter, 2,9,12,32 these calculations have not been done in this study.

From Fig. 6 it can be seen that ${ }^{119} \mathrm{Sb}$ has the highest TND values of the isotopes tested for both nuclear, cytoplasmic, and membrane bound activity distributions. For the nuclear distribution ${ }^{119} \mathrm{Sb}$ is closely followed by ${ }^{125} \mathrm{I}$ and ${ }^{165} \mathrm{Er}$. However, the long half-life $\left(\mathrm{T}_{1 / 2}=59.4 \mathrm{~d}\right)$ of ${ }^{125} \mathrm{I}$ is a drawback in rapid proliferating cells as reported by ODonoghue and Wheldon $^{33}$ in combination with the resulting thyroidal uptake of this isotope if in vivo deiodination occurs.

The use of ${ }^{165} \mathrm{Er}$ for targeted radiotherapy has been suggested recently by Beyer et al. $^{34}$ Even though it has a $\mathrm{p} / \mathrm{e}$ ratio of 4.7 caused by the abundant X-ray emission, and the dose delivered to the cell nucleus per ${ }^{165} \mathrm{Er}$ disintegration is quite low compared to the other radionuclides tested, it has a high TND. For comparison, the $p / e$ ratio for ${ }^{119} \mathrm{Sb}$ is 0.9 (the value of 0.09 reported by Bernhardt et al. ${ }^{29}$ must be a misprint). Thus, ${ }^{165} \mathrm{Er}$ may be another promising radionuclide for therapy.

Still, of all the radionuclides considered in this study, ${ }^{119} \mathrm{Sb}$ seems to be the most promising radionuclide for tar- geted radiotherapy of small tumors, micrometastases, or single cancer cells. This is in good agreement with the theoretical study of Bernhardt et al. ${ }^{29}$ Using predefined criteria and, as described above, assuming the tumor to be a sphere and the body an ellipsoid, both with uniform activity distributions, five isotopes were found suitable for targeted radiotherapy of small tumors, among them ${ }^{119} \mathrm{Sb}$.

\section{III.B. ${ }^{117}$ Sb SPECT}

Internal dosimetry constitutes a very important part of radionuclide therapy but because of the absence of penetrating photon emission from the ${ }^{119} \mathrm{Sb}$ decay, the time-activity curves and normal-tissue uptake will be difficult to determine from this radionuclide - the same effect as observed with ${ }^{90} \mathrm{Y} .{ }^{16}$ However, by a tracer study with SPECT using the isotope ${ }^{117} \mathrm{Sb}$, it should be possible to partly overcome this. ${ }^{117} \mathrm{Sb}$ decays mainly by EC (only $1.7 \% \beta^{+}$) with the emission of the nearly single $\gamma$-ray of $158.56 \mathrm{keV}$ suitable for imaging. In fact, the energies and intensities of the emitted photons in the ${ }^{117} \mathrm{Sb}$ decay are very similar to the photons emitted by the widely used SPECT isotope ${ }^{123} \mathrm{I}$ (see Table II).

${ }^{117} \mathrm{Sb}$ can be produced and used for labeling a precursor using the same technique as for ${ }^{119} \mathrm{Sb}$. Both isotopes being of the same element also ensures identical properties in vivo. In this respect, the isotope pair ${ }^{117} \mathrm{Sb} /{ }^{119} \mathrm{Sb}$ resembles the ${ }^{86} \mathrm{Y} /{ }^{90} \mathrm{Y}$ pair.

From the planar scintigraphy and the transversal slice (corresponding to $1.9 \mathrm{~cm}$ ) through the SPECT tomography of the Jaszczak phantom (Fig. 7), it can be seen that ${ }^{117} \mathrm{Sb}$ is a suitable imaging isotope. It was possible to locate both the hot-spot and the three largest spherical cold-spots $(\varnothing 38 \mathrm{~mm}$, $\varnothing 31.8 \mathrm{~mm}$, and $\varnothing 25.4 \mathrm{~mm}$ ) in the SPECT scan and all the cold-rods were visible in the planar scintigraphy. The coldrods were not visible in the SPECT scan, though, but this was primarily due to a too low number of counts in the separate frames.

\section{III.C. ${ }^{119} \mathrm{Sb}$ and ${ }^{117} \mathrm{Sb}$ production yields}

The ${ }^{119} \mathrm{Sb}$ and ${ }^{117} \mathrm{Sb}$ irradiation yields at the end of bombardment (EOB) were measured to be $1.85 \pm 0.12 \mathrm{MBq} / \mu \mathrm{Ah}$ and $34.6 \pm 0.8 \mathrm{MBq} / \mu \mathrm{Ah}$, respectively, using the irradiation conditions and target thicknesses described in Sec. II B. Based on these measurements and assuming the use of proper water cooling of the enriched tin target, one should be able to produce approximately $4.4 \mathrm{GBq}$ of ${ }^{119} \mathrm{Sb}$ at EOB in $8 \mathrm{~h}$ with a low energy cyclotron. This is with an electroplated target of thickness $50 \mathrm{mg} / \mathrm{cm}^{2}$ in a

TABLE II. Nuclear data for ${ }^{117} \mathrm{Sb}$ and ${ }^{123} \mathrm{I}$. Only $\gamma$-photons with intensities above $0.3 \%$ are shown (Ref. 27).

\begin{tabular}{lrllll}
\hline \hline \multicolumn{1}{c}{ Isotope } & Half-life & Decay mode & \multicolumn{3}{c}{ Major $\gamma$-lines in keV (abundance) } \\
\hline${ }^{117} \mathrm{Sb}$ & $2.80 \mathrm{~h}$ & EC, $1.7 \% \beta^{+}$ & $158.56(86 \%)$ & $511(3.4 \%)$ & $861.35(0.31 \%)$ \\
${ }^{123} \mathrm{I}$ & $13.27 \mathrm{~h}$ & EC & $158.97(83 \%)$ & $528.96(1.39 \%)$ & $440.02(0.43 \%)$ \\
& & & $538.54(0.38 \%)$ & $505.33(0.32 \%)$ & \\
\hline \hline
\end{tabular}



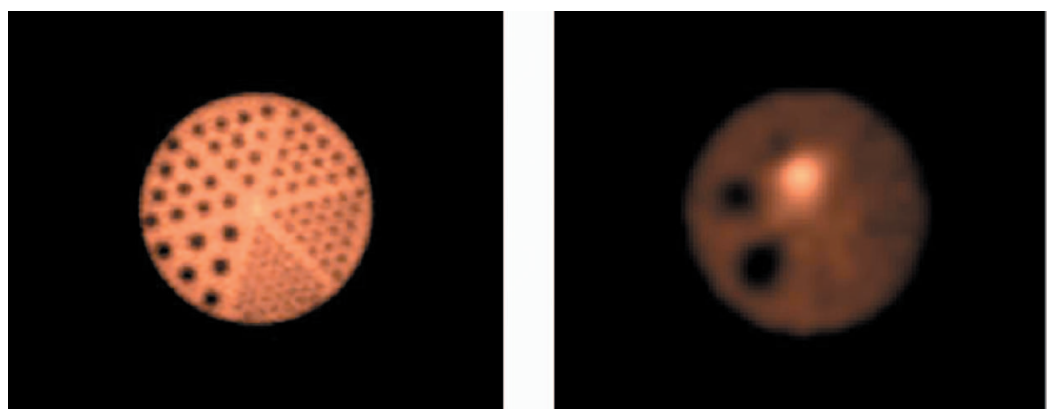

FIG. 7. The planar scintigraphy showing the cold rods (left) and a transversal slice through the SPECT tomography showing the hot spot and the three largest cold spheres (right) in the Jaszczak phantom using ${ }^{117} \mathrm{Sb}$. The planar scintigraphy was acquired with the phantom standing on its end on top of the collimator.

standard $90^{\circ}$ target/beam geometry using a collimated $(\varnothing 10 \mathrm{~mm}) 11.0 \mathrm{MeV}$ proton beam and a beam current of $35 \mu \mathrm{A}$. In this extrapolation, we have assumed that the nuclear cross sections for the ${ }^{119} \mathrm{Sn}(\mathrm{p}, \mathrm{n}){ }^{119} \mathrm{Sb}$ reaction are constant in the energy interval (11.0-9.9 MeV) represented by the $50 \mathrm{mg} / \mathrm{cm}^{2}$ target thickness. On the other hand, by considering the reaction threshold energy of $11.0 \mathrm{MeV}$ for the ${ }^{119} \mathrm{Sn}(\mathrm{p}, 2 \mathrm{n}){ }^{118} \mathrm{Sb}$ reaction, ${ }^{35}$ one would expect an increase in the cross sections above the $10.1 \mathrm{MeV}$ proton energy that was used in our yield measurement for the ${ }^{119} \mathrm{Sn}(\mathrm{p}, \mathrm{n}){ }^{119} \mathrm{Sb}$ reaction. This means that the extrapolated production yield may be an underestimate of the real obtainable yield using the larger amount of enriched target material than what was used in this study.

If even higher activities are needed, this can be achieved by either increasing the irradiation time or by a further increase in the target thickness. Another approach is to use an inclined target/beam geometry to allow a high increase in the beam current resulting in a considerable increase in the obtainable yield. Such high current irradiations $(\sim 200 \mu \mathrm{A})$ on $a{ }^{\text {nat }}$ Sn target using a dedicated PET cyclotron have recently been performed in our department (unpublished data) demonstrating the capability of producing several tens of GBqs of therapeutic isotopes locally. Hence, it will be possible to produce the amounts of ${ }^{119} \mathrm{Sb}$ radioactivity that is required for initial patient studies and clinical trials in radionuclide therapy using a small medical cyclotron.

The shorter half-life of ${ }^{117} \mathrm{Sb}$ and the need for a much smaller amount of radioactivity for each patient in a diagnostic study than for therapy, makes the ${ }^{117} \mathrm{Sb}$ production less demanding than the ${ }^{119} \mathrm{Sb}$ production. Using the same extrapolation conditions as above, it will be possible to produce approximately $23 \mathrm{GBq}$ of ${ }^{117} \mathrm{Sb}$ at EOB in a $4 \mathrm{~h}$ irradiation, which should be sufficient for several patient scans.

\section{CONCLUSION}

Based on theoretical dosimetry calculations for different subcellular distributions of several Auger-electron emitting isotopes, we have identified ${ }^{119} \mathrm{Sb}$ as being a potent radionuclide for targeted radiotherapy of small tumors, micrometastases, and single cancer cells. Using the MIRD-model, we have calculated the cellular $\mathrm{S}$-values for this isotope. In addition, we have measured the proton irradiation yields for the production of ${ }^{119} \mathrm{Sb}$ and the corresponding imaging isotope ${ }^{117} \mathrm{Sb}$ using a low-energy cyclotron. From a planar scintigraphy and a SPECT scan of a Jaszczak phantom, we have shown that the latter isotope is well suited for SPECT-based patient-specific dosimetry of an ${ }^{119} \mathrm{Sb}$-labeled radiopharmaceutical. The production yields show that it will be possible to produce both isotopes with a standard PET-cyclotron in sufficient quantities for patient imaging and therapy.

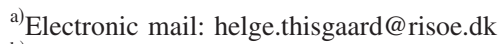

${ }^{b)}$ Author to whom correspondence should be addressed. Electronic mail: mikael.jensen@risoe.dk

${ }^{1}$ E. Janson et al., "Nuclear localization of In-111 after intravenous injection of [In-111-DTPA-D-Phe(1)]-octreotide in patients with neuroendocrine tumors," J. Nucl. Med. 41, 1514-1518 (2000).

${ }^{2}$ A. Capello et al., "Peptide receptor radionuclide therapy in vitro using [In-111-DTPA(0)]octreotide," J. Nucl. Med. 44, 98-104 (2003).

${ }^{3}$ R. Michel, M. Brechbiel, and M. Mattes, "A comparison of 4 radionuclides conjugated to antibodies for single-cell kill,” J. Nucl. Med. 44, 632-640 (2003).

${ }^{4} \mathrm{~T}$. Behr et al., "Therapeutic advantages of Auger electron- over betaemitting radiometals or radioiodine when conjugated to internalizing antibodies," Eur. J. Nucl. Med. 27, 753-765 (2000).

${ }^{5} \mathrm{R}$. O'Donnell, "Nuclear localizing sequences: An innovative way to improve targeted radiotherapy," J. Nucl. Med. 47, 738-739 (2006).

${ }^{6} \mathrm{P}$. Chen et al., "Nuclear localizing sequences promote nuclear translocation and enhance the radiotoxicity of the anti-CD33 monoclonal antibody HuM195 labeled with In-111 in human myeloid leukemia cells," J. Nucl. Med. 47, 827-836 (2006).

${ }^{7}$ D. L. Costantini et al., "111In-labeled Trastuzumab (Herceptin) modified with nuclear localization sequences (NLS): An auger electron-emitting radiotherapeutic agent for HER2/neu-amplified breast cancer," J. Nucl. Med. 48, 1357-1368 (2007).

${ }^{8}$ R. Howell, "Radiation spectra for Auger-electron emitting radionuclides-Report No. 2 of Aapm-Nuclear-Medicine-Task-Group No. 6," Med. Phys. 19, 1371-1383 (1992).

${ }^{9}$ A. Kassis, "Cancer therapy with Auger electrons: Are we almost there?," J. Nucl. Med. 44, 1479-1481 (2003).

${ }^{10} \mathrm{~S}$. Govindan et al., "Radionuclides linked to a CD74 antibody as therapeutic agents for B-cell lymphoma: Comparison of Auger electron emitters with beta-particle emitters," J. Nucl. Med. 41, 2089-2097 (2000).

${ }^{11}$ A. Kassis, "The amazing world of Auger electrons," Int. J. Radiat. Biol. 80, 789-803 (2004).

${ }^{12} \mathrm{~S}$. Adelstein et al., "Radiotoxicity of iodine-125 and other Augerelectron-emitting radionuclides: Background to therapy," Cancer Biother. Radiopharm. 18, 301-316 (2003).

${ }^{13} \mathrm{R}$. Howell and A. Bishayee, "Bystander effects caused by nonuniform distributions of DNA-incorporated I-125," Micron 33, 127-132 (2002).

${ }^{14} \mathrm{G}$. Griffiths et al., "Cytotoxicity with Auger electron-emitting radionuclides delivered by antibodies," Int. J. Cancer 81, 985-992 (1999).

${ }^{15} \mathrm{M}$. Mattes, "Radionuclide-antibody conjugates for single-cell cytotoxicity," Cancer 94, 1215-1223 (2002).

${ }^{16} \mathrm{~F}$. Rosch et al., "Uptake kinetics of the somatostatin receptor ligand [Y-86] DOTA-DPhe(1)-Tyr(3)-octreotide ([Y-86] SMT487) using positron emission tomography in non-human primates and calculation of radiation doses of the Y-90-labelled analogue," Eur. J. Nucl. Med. 26, 358366 (1999)

${ }^{17} \mathrm{H}$. Herzog et al., "Measurement of pharmacokinetics of yttrium-86 radiopharmaceuticals with pet and radiation-dose calculation of analogous 
Y-90 radiotherapeutics,” J. Nucl. Med. 34, 2222-2226 (1993).

${ }^{18}$ A. Helisch et al., "Pre-therapeutic dosimetry and biodistribution of Y-86DOTA-Phe(1)-Tyr(3)-octreotide versus In-111-pentetreotide in patients with advanced neuroendocrine tumours," Eur. J. Nucl. Med. Mol. Imaging 31, 1386-1392 (2004).

${ }^{19} \mathrm{~K}$. Kolbert et al., "Prediction of absorbed dose to normal organs in thyroid cancer patients treated with I-131 by use of I-124 PET and 3-dimensional internal dosimetry software," J. Nucl. Med. 48, 143-149 (2007).

${ }^{20} \mathrm{G}$. Sgouros et al., "Patient-specific dosimetry for I-131 thyroid cancer therapy using I-124 PET and 3-dimensional-internal dosimetry (3D-ID) software," J. Nucl. Med. 45, 1366-1372 (2004).

${ }^{21} \mathrm{~S}$. Goddu et al., MIRD Cellular S Values (Society of Nuclear Medicine, Reston, Virginia, 1997).

${ }^{22}$ M. J. Berger, "Beta-ray dosimetry calculations with the use of point kernels," Medical Radionuclides: Radiation Dose and Effects, AEC Symposium Series No. 20, edited by R. J. Cloutier, C. L. Edwards, and W. S. Snyder, US Atomic Energy Commission, pp. 63-86 (1970).

${ }^{23} \mathrm{~A}$. Cole, "Absorption of 20-Ev to 50000 -Ev electron beams in air and plastic,” Radiat. Res. 38, 7-33 (1969).

${ }^{24 " N u c l e a r ~ D e c a y ~ D a t a ~ F i l e s ~ f o r ~ D o s e ~ C a l c u l a t i o n s " ~(2001) ~}$

${ }^{25}$ "The RAdiation dose assessment resource-RADAR," http:// www.doseinfo-radar.com/ (2005).
26،IAEA charged-particle cross section database for medical radioisotope production," http://www-nds.iaea.or.at/medical/ (2000).

27"WWW table of radioactive isotopes," http://ie.lbl.gov/toi/ (2004).

${ }^{28} \mathrm{~K}$. Sastry et al., "Electron dosimetry for radioimmunotherapy-Optimal electron-energy," Radiat. Prot. Dosim. 13, 249-252 (1985).

${ }^{29}$ P. Bernhardt et al., "Low-energy electron emitters for targeted radiotherapy of small tumours," Acta Oncol. 40, 602-608 (2001).

${ }^{30} \mathrm{P}$. Zanzonico, "Invited commentary-Model-based versus patient-specific dosimetry: Blurring the lines," J. Nucl. Med. 43, 1665-1666 (2002).

${ }^{31}$ S. Goddu, D. Rao, and R. Howell, "Multicellular dosimetry for micrometastases-Dependence of self-dose versus cross-dose to cellnuclei on type and energy of radiation and subcellular-distribution of radionuclides," J. Nucl. Med. 35, 521-530 (1994).

${ }^{32} \mathrm{~A}$. Kassis and S. Adelstein, "Radiobiologic principles in radionuclide therapy," J. Nucl. Med. 46, 4S-12S (2005).

${ }^{33} \mathrm{~J}$. ODonoghue and T. Wheldon, "Targeted radiotherapy using Auger electron emitters," Phys. Med. Biol. 41, 1973-1992 (1996).

${ }^{34}$ G. Beyer, S. Zeisler, and D. Becker, "The Auger-electron emitter Er-165: Excitation function of the Ho-165(p, n)Er-165 process," Radiochim. Acta 92, 219-222 (2004).

35“"Q-value calculator," National Nuclear Data Center, Brookhaven National Laboratory, http://www.nndc.bnl.gov/qcalc/ (2007). 